\title{
Solution strategies for 1D elastic continuum with long-range interactions: Smooth and fractional decay
}

\author{
G. Failla ${ }^{a, *}$, A. Santini ${ }^{a}$, M. Zingales ${ }^{b}$ \\ a Dipartimento di Meccanica e Materiali (MECMAT), University of Reggio Calabria, Via Graziella, 89122 Reggio Calabria, Italy \\ ${ }^{\mathrm{b}}$ Dipartimento di Ingegneria Strutturale, Aerospaziale e Geotecnica (DISAG), University of Palermo, Viale delle Scienze, 90128 Palermo, Italy
}

\section{A R T I C L E I N F O}

\section{Article history:}

Received 6 November 2008

Received in revised form 8 July 2009

Available online 2 October 2009

\section{Keywords:}

Non-local elasticity

Long-range interactions

Weak formulation of elastic problems

Fractional calculus

Fractional finite differences

\begin{abstract}
A B S T R A C T
An elastic continuum model with long-range forces is addressed in this study within the context of approximate analytical methods. Such a model stems from a mechanically-based approach to non-local theory where long-range central forces are introduced between non-adjacent volume elements. Specifically, long-range forces depend on the relative displacement, on the volume product between interacting elements and they are proportional to a proper, material-dependent, distance-decaying function. Smooth-decay functions lead to integro-differential governing equations whereas hypersingular, fractional-decay functions lead to a fractional differential governing equation of Marchaud type. In this paper the Galerkin and the Rayleigh-Ritz method are used to build approximate solutions to the integro-differential and the fractional differential governing equations. Numerical applications show the accuracy of the proposed approximate solutions as compared to the finite difference approximation and to the fractional finite difference approximation.
\end{abstract}

(c) 2009 Elsevier Ltd. All rights reserved.

\section{Introduction}

In the last decades experimental results which cannot be explained by classical local continuum mechanics have forced scientists and engineers to formulate alternative theories which may better fit to observed phenomena. These include shear bands in tensile specimens, acoustic wave dispersion in granular materials, softening phenomena and smoothing effects of concentrated stress at crack tips.

It is generally believed that most of these phenomena may be given an exhaustive explanation at a microstructural level and, since the end of the 1950s, a first approach to this problem has been then framed in the context of atomic theory and lattice mechanics. However, the strong computational effort involved by the use of such theories has soon motivated an increasing interest towards continuum formulations where microstructural effects are accounted for by properly modified constitutive relations, including non-local terms as weighted integrals (Kroner, 1967; Eringen, 1972) or gradients (Aifantis, 1994; Polizzotto, 2003). These approaches enjoy the beauty of a constitutive relation that does not involve additional state variables of the elastic problem. On the other hand, approaches involving non-local weighted integrals lead to non-convex potential energy functions, while approaches involving non-local gradients may experience inherent difficulties

\footnotetext{
* Corresponding author. Tel.: +39 0965875215; fax: +39 0965875201

E-mail address: giuseppe.failla@unirc.it (G. Failla).
}

in deriving the boundary conditions. As an alternative to modified constitutive relations, microstructural effects have been accounted for in the equilibrium equation (i) by including non-local forces in an integral form (Silling, 2000; Silling et al., 2003); (ii) by the socalled continualization, where a continuum model is built based on higher-order differential operators ensuring that the continuum behaviour approximates the behaviour of the discrete lattice (Metrikine and Askes, 2002a,b; Andrianov and Awrejcewicz, 2005).

Recently, a non-local continuum model has been proposed for a 1D bar using a mechanically-based approach (Di Paola and Zingales, 2008; Di Paola et al., 2009). The 1D bar has been modelled as the continuum limit of a discrete ensemble of distinct volume elements. Adjacent elements exert mutual classical contact forces while non-adjacent elements exert mutual, distance-decaying, central forces. The latter are taken to be proportional to the interacting volumes and to their relative displacement. As the size of the volume elements reduces to zero, the resulting 1D continuum is found to be governed by an integro-differential equation for long-range forces with a smooth decay or by a fractional differential equation for long-range forces with a fractional decay. In both cases either mechanical or kinematic boundary conditions may be consistently enforced. It may be also shown that the mechanicallybased approach enjoys all the features of the elasticity theory since the associated elastic potential energy is convex and positive definite.

The solution to the integro-differential equation has been built based on a standard finite difference approximation (FDA), 
suggested by the underlying physical model of distinct volume elements itself (Di Paola et al., 2009). Similarly, a fractional finite difference approximation (FFDA) (Shkanukov, 1996) has been adopted to solve the fractional differential equation (Di Paola and Zingales, 2008). However, in both cases all the advantages of the continuum representation are lost since, depending on the problem at hand, a relevant number of volume elements may be required to attain convergence and at the expense, obviously, of an increasing computational effort. This may be typically the case not only of statics but also of dynamics applications where, for instance, building the eigensolution of large size matrices does involve significant computational costs.

In this study the authors aim to show that the solution for the proposed non-local bar, when long-range forces feature either a smooth or a fractional decay, may be built by well-known approximate methods of classical elasticity theory, such as the Galerkin method and the Rayleigh-Ritz method, both formulated based on variational principles. The results obtained prove that accurate solutions for the proposed non-local bar may be built in a continuum setting, without resorting to back-discretization of the governing equations. Also, it is seen that the two methods lead to the same governing equations yet following a different approach, proving that the operators are self-adjoint and a convex elastic potential energy function is associated to the proposed non-local model.

The mechanics of the proposed non-local bar is described in Section 2. The variational formulation of the governing equations, including the derivation of pertinent boundary conditions, is presented in Section 3. Also, in Section 3 it is shown that the proposed model of non-local elasticity is thermodynamically consistent and that no thermodynamic restrictions hold on the derived boundary conditions. Then the formulation of the Galerkin method and the Rayleigh-Ritz method to solve the elastic problem formulated in Section 2 is given in Section 4. Numerical results are presented in Section 5, where the FDA and the FFDA are used as benchmark solutions. For completeness, in the Appendix some final remarks about the relation between the proposed model of non-local bar and the well-known Eringen theory are reported.

\section{A model of 1D elastic continuum with long-range forces}

The fundamental concepts are introduced considering the simple bar in Fig. 1. Be $x$ an axis coinciding with the geometrical axis of the bar and positive rightward. Be $L$ the length, $E$ and $A$ the Young modulus and the cross-sectional area, respectively; also, be $u(x)$ the axial displacement and $f(x)$ the external force per unit volume, both positive rightward.

Let us introduce a discrete model of the bar into $n$ small finite volumes $V_{j}=A \Delta x$, where $n=L / \Delta x$. Any volume $V_{j}$ is in equilibrium under the external body force $f_{j} A \Delta x$, where $f_{j}=f\left(x_{j}\right)$, being $x_{j}=(2 j-1) \Delta x / 2(j=1,2, \ldots, n), N_{j-1}$ and $N_{j}$ are the axial contact forces exerted by the adjacent volumes $V_{j-1}$ and $V_{j+1}$, respectively. Further, within the theoretical framework of continuum mechanics with long-range forces, it may be consistently assumed that any volume $V_{j}$ is acted upon by the resultant $Q_{j}$ of long-range forces due to surrounding, non-adjacent volume elements. Specifically, $Q_{j}$ is taken as the resultant of long-range central forces applied to the centroid of the volume elements (see Fig. 1), given by:

$Q_{j}=\sum_{h=j+1}^{n} Q^{(h \cdot j)}+\sum_{h=1}^{j-1} Q^{(h \cdot j)}$,

where $Q^{(h, j)}=q^{(h . j)} V_{h} V_{j}$, for $q^{(h, j)}$ given as

$q^{(h, j)}=\left[u\left(x_{h}\right)-u\left(x_{j}\right)\right] g\left(x_{h}, x_{j}\right)$.

In Eq. (2) $g\left(x_{h}, x_{j}\right)=g\left(\left|x_{h}-x_{j}\right|\right)$ is an appropriate material-dependent and distance-decaying positive function, taken to be monotonically decreasing.

The static equilibrium equation of the volume element $V_{j}$ in Fig. 1 is then written as

$$
\begin{aligned}
\Delta N_{j}+Q_{j}+f_{j} A \Delta x= & \Delta N_{j}+\sum_{h=j+1}^{n} q^{(h, j)}(A \Delta x)^{2} \\
& +\sum_{h=1}^{j-1} q^{(h, j)}(A \Delta x)^{2}+f_{j} A \Delta x=0,
\end{aligned}
$$

where $\Delta N=N_{j}-N_{j-1}$. Dividing Eq. (3) by $A \Delta x$ and letting $\Delta x \rightarrow 0$ yields the equilibrium equation of the $1 \mathrm{D}$ non-local bar in the form

$$
\frac{d \sigma_{l}(x)}{d x}+A \int_{0}^{L} q(x, \xi) d \xi=-f(x), \quad \text { for } q(x, \xi)=g(x, \xi) \eta(x, \xi),
$$

where $\eta(x, \xi)=u(\xi)-u(x) ; \sigma_{l}(x)=N(x) / A$ is the local stress due to the contact forces (i.e., the Cauchy stress) and the integral terms in Eq. (4) are the resultants (per unit volume) of the long-range forces due, respectively, to the volume elements to the right and to the left of the location $x$. Based on the resultant non-local stress $\sigma_{n l}(x)$, given as

$\sigma_{n l}(x)=A \int_{\xi=x}^{L} \int_{\varsigma=0}^{x} q(\xi, \varsigma) d \xi d \varsigma$

it may be proved that Eq. (4) reverts to

$\frac{d \sigma(x)}{d x}=\frac{d \sigma_{l}(x)}{d x}+\frac{d \sigma_{n l}(x)}{d x}=-f(x)$,

where $\sigma(x)=\sigma_{l}(x)+\sigma_{n l}(x)$ is the overall axial stress. In passing it has to be remarked that the resultant non-local stress in Eq. (5) is obtained by cutting the bar into two parts and evaluating the resultant of the long-range forces exchanged by the volume elements to the right and to the left of the location $x$.

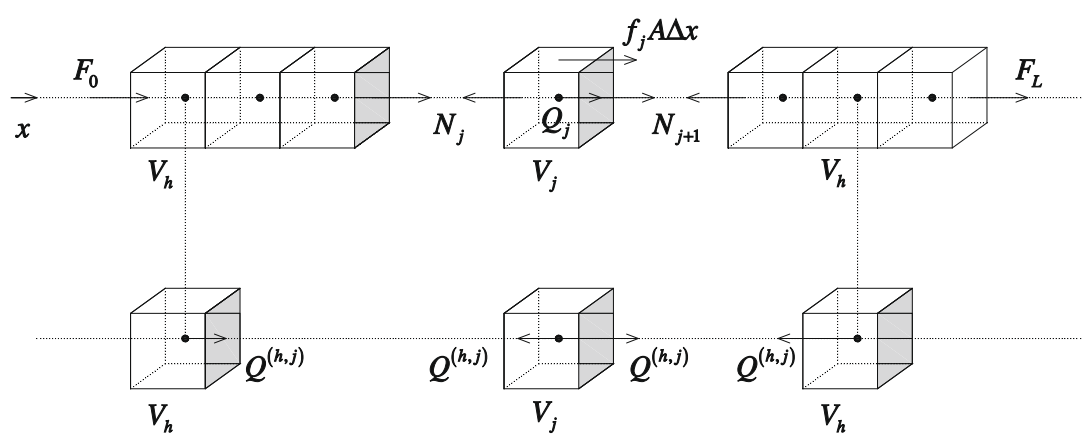

Fig. 1. (a) Discretized elastic bar loaded by an external volume force field $f(x)$; (b) Equilibrium of the volume element $V_{j}$. 
The solution for the non-local bar is sought based on the equilibrium equation (6) along with the following equations:

$$
\left\{\begin{array}{l}
\frac{d \sigma(x)}{d x}=\frac{d \sigma_{l}(x)}{d x}+A \int_{0}^{L} q(x, \xi) d \xi=-f(x) \\
\frac{d u(x)}{d x}=\varepsilon(x) ; \quad \eta(x, \xi)=u(\xi)-u(x) \\
\sigma(x)=\sigma_{l}(x)+\sigma_{n l}(x) \\
\quad=\bar{E}(x) \varepsilon(x)+A \int_{\xi=x}^{L} \int_{\zeta=0}^{x} g(\xi, \varsigma)[u(\xi)-u(\varsigma)] d \xi d \varsigma .
\end{array}\right.
$$

The reason for which equation $\eta(x, \xi)=u(\xi)-u(x)$ can be consistently considered among the equations governing the non-local bar will appear more clear in the following developments in Section 3 , where the governing equations will be derived within a variational framework. Also, note that in Eq. (7d) the constitutive equation for the local stress $\sigma_{l}(x)$ in terms of the strain $\varepsilon(x)$ is taken to be $\sigma_{l}(x)=\bar{E} \varepsilon(x)$, for $\bar{E}=\beta_{1} E$, being $\beta_{1}$ a positive dimensionless constant introduced, as in the integral model of non-local elasticity (Fuschi and Pisano, 2003), to weigh the amount of local stress. The latter equation (7d) represents the constitutive equation relating the local and non-local contribution to the overall stress. Also it is to be observed that the contribution provided by the long-range forces at the location $x$ is in integral form. This consideration is necessary since it is used to specify the static boundary conditions associated to the elastic problem. Note in fact that the boundary conditions for the non-local bar are given as

$u(0)=u_{0} ; \quad u(L)=u_{L}$,

$\sigma(0) A=\sigma_{l}(0) A=-F_{0} ; \quad \sigma(L) A=\sigma_{l}(L) A=F_{L}$,

where $F_{0}$ and $F_{L}$ are the end forces at $x=0$ and $x=L$, respectively; further, recognize that in Eq. (9) $\sigma_{n l}(0)=\sigma_{n l}(L)=0$, as it may be derived from Eq. (7c).

Based on Eq. (7), the equilibrium equation may be rewritten in terms of displacement as

$\bar{E} \frac{d^{2} u(x)}{d x^{2}}+A \int_{0}^{L} g(x, \xi)[u(\xi)-u(x)] d \xi=-f(x)$.

For completeness it is noted that, based on the same reasoning followed above, Eq. (10) generalizes for an infinite bar in the form

$\bar{E} \frac{d^{2} u(x)}{d x^{2}}+A \int_{-\infty}^{\infty} g(x, \xi)[u(\xi)-u(x)] d \xi=-f(x)$.

It is now worth pointing out that Eqs. (10) and (11) involve an integral term analogous to the integral term accounting for longrange forces in a peridynamic bar (Silling et al., 2003). However Eqs. (10) and (11) are integro-differential equations and, due to the differential term, they yield continuous displacement fields under concentrated forces. This is not the case in the peridynamic solution (Silling et al., 2003).

Some interesting remarks on the relation between the proposed model and the well-known Eringen theory of non-local elasticity (Eringen, 1972) are given, in more details, in the Appendix.

Next some further comments are made about the specific functional class to adopt for the distance-decaying function $g(|x-\xi|)$. It may be chosen as a smooth function without singularities (like Gaussian-type or continuous with an isolated discontinuity like the exponential decay) so that Eq. (10) is an integro-differential equation. On the other hand an attractive choice for the distance decaying function is related to the fractional power-law decay

$g(|x-\xi|)=\frac{\beta_{2} E \alpha}{A^{2} \Gamma(1-\alpha)} \frac{c_{\alpha}}{|x-\xi|^{1+\alpha}}$,

which may prove appropriate for materials involving long-range interactions at a molecular level such as electrostatic, long-range elastic bonds in next to the nearest next (NNN) lattices or magnetic forces as Lorentz forces. More details about such a power-law decay of the long-range interactions may be found in very recent literature (Laskin and Zaslavsky, 2006). Specifically, in Eq. (12) $\Gamma(\cdot)$ is the Euler-Gamma function; $\alpha$ is a real, material-dependent parameter ruling the decay of the interactions, being $0 \leqslant \alpha \leqslant 1 ; c_{\alpha}$ is a dimensional coefficient of fractional order, $\left[c_{\alpha}\right]=L^{\alpha}$ also depending on the material; $\beta_{2}=1-\beta_{1}$ is a positive dimensionless constant introduced, as in the integral model of non-local elasticity (Fuschi and Pisano, 2003), to weigh the amount of non-local stress. This kind of distance-decaying function, if replaced for $g(|x-\xi|)$ into Eq. (11), yields an hypersingular kernel that coincides with the sum of Marchaud left and right fractional derivatives, leading to the second-order fractional differential equation (Di Paola and Zingales, 2008)

$\bar{E} \frac{d^{2} u(x)}{d x^{2}}-C_{\alpha}\left[\left(\mathbf{D}_{-}^{\alpha} u\right)(x)+\left(\mathbf{D}_{+}^{\alpha} u\right)(x)\right]=-f(x)$,

where $C_{\alpha}=\beta_{2} E c_{\alpha} / A^{2}$. In Eq. (13), the left and right Marchaud fractional derivatives are defined as the convolution integrals (for details about fractional calculus see Samko et al., 1988)

$$
\begin{aligned}
& \left(\mathbf{D}_{-}^{\alpha} u\right)(x)=\frac{\alpha}{\Gamma(1-\alpha)} \int_{-\infty}^{x} \frac{u(x)-u(\xi)}{(x-\xi)^{1+\alpha}} d \xi \\
& \left(\mathbf{D}_{+}^{\alpha} u\right)(x)=\frac{\alpha}{\Gamma(1-\alpha)} \int_{x}^{\infty} \frac{u(x)-u(\xi)}{(\xi-x)^{1+\alpha}} d \xi
\end{aligned}
$$

For a finite domain such an equivalence between hypersingular kernels and fractional operators does not hold and the governing equation of the axial displacement is obtained in terms of the truncated Marchaud operators in the form

$\bar{E} \frac{d^{2} u(x)}{d x^{2}}-C_{\alpha}\left[\left(\widehat{\mathbf{D}}_{0^{+}}^{\alpha} u\right)(x)+\left(\widehat{\mathbf{D}}_{L^{-}}^{\alpha} u\right)(x)\right]=-f(x)$,

where the truncated Marchaud operator is related to the integral terms in the Marchaud fractional derivative on a finite domain, defined as

$$
\begin{aligned}
& \left(\mathbf{D}_{0^{+}}^{\alpha} u\right)(x)=\frac{\alpha}{\Gamma(1-\alpha)} \int_{0}^{x} \frac{u(x)-u(\xi)}{(x-\xi)^{1+\alpha}} d \xi+\frac{1}{\Gamma(1-\alpha)} \frac{u(x)}{x^{\alpha}}=\left(\widehat{\mathbf{D}}_{0^{+}}^{\alpha} u\right)(x)+\frac{1}{\Gamma(1-\alpha)} \frac{u(x)}{x^{\alpha}} \\
& \left(\mathbf{D}_{+}^{\alpha} u\right)(x)=\frac{\alpha}{\Gamma(1-\alpha)} \int_{x}^{L} \frac{u(x)-u(\xi)}{(\xi-x)^{1+\alpha}} d \xi+\frac{1}{\Gamma(1-\alpha)} \frac{u(x)}{(L-x)^{\alpha}}=\left(\widehat{\mathbf{D}}_{L^{-}}^{\alpha} u\right)(x)+\frac{1}{\Gamma(1-\alpha)} \frac{u(x)}{(L-x)^{\alpha}} .
\end{aligned}
$$

The non-integral terms in Eq. (16a,b) represent additional restraints that are not included in the model and that are not present in the infinite domain (see also Cottone et al., 2009).

\section{Variational formulation to the proposed 1D elastic continuum}

Next it will be shown that the equations governing the proposed non-local bar, including the boundary conditions, may be derived within a consistent variational framework. Also, based on the well-established procedure by Polizzotto (for details see Polizzotto, 2001), the thermodynamic consistency of the model will be proved.

\subsection{Total potential energy functional}

The total potential energy functional can be cast in the following form

$$
\begin{aligned}
\Phi(u, \varepsilon, \eta)= & \frac{1}{2}\left\{\int_{0}^{L} \bar{E} A \varepsilon(x)^{2} d x+\frac{A^{2}}{2} \int_{0}^{L} \int_{0}^{L} g(x, \xi) \eta(x, \xi)^{2} d \xi d x\right\} \\
& -\left[u(0) F_{0}+u(L) F_{L}+A \int_{0}^{L} f(x) u(x) d x\right],
\end{aligned}
$$


where $u(x), \varepsilon(x)$ and $\eta(x, \xi)$ are kinematically admissible, that is they satisfy Eq. (7b,c). The first two integral terms in the r.h.s. represent the elastic potential energy of the solid, including the well-known elastic potential energy due to the contact forces and defined in terms of the state variable $\varepsilon(x)$ and the non-local elastic potential energy due to the long-range forces and defined over the space of the relative displacement function $\eta(x, \xi)=u(\xi)-u(x)$, i.e. the kinematic state variable associated to the long-range force $q(x, \xi)$ introduced, on a mechanical basis, in Section 2. Note also that the elastic potential energy of the solid is a quadratic form of the state variables and positive definite under the assumption of positive distance-decaying function.

It can be seen that the Euler-Lagrange equations associated to Eq. (17) and the corresponding natural boundary conditions coincide, respectively, with the equations reported in Section 2 on a mechanical basis. To this purpose note that

$$
\begin{aligned}
\delta \Phi(u, \varepsilon, \eta)= & \frac{\partial \Phi}{\partial \varepsilon} \delta \varepsilon+\frac{\partial \Phi}{\partial \eta} \delta \eta \\
= & \int_{0}^{L} \sigma_{l} A \cdot \delta \varepsilon d x+\frac{1}{2} \int_{0}^{L} \int_{0}^{L} A^{2} \cdot g(x, \xi) \eta \cdot \delta \eta d x d \xi \\
& -\left[F_{0} \delta u(0)+F_{L} \delta u(L)+\int_{0}^{L} f A \delta u d x\right]
\end{aligned}
$$

Eq. (18) may be further simplified since

$$
\int_{0}^{L} \sigma_{l} A \cdot \delta \varepsilon d x=-\int_{0}^{L} \frac{d \sigma_{l}}{d x} A \cdot \delta u d x+\sigma_{l}(L) A \cdot \delta u(L)-\sigma_{l}(0) A \cdot \delta u(0)
$$

and

$\frac{1}{2} \int_{0}^{L} \int_{0}^{L} A^{2} g(x, \xi) \eta \cdot \delta \eta d x d \xi$

$$
\begin{aligned}
= & \frac{1}{2} \int_{0}^{L}\left[\int_{0}^{L} A^{2} g(x, \xi) \eta d x\right] \delta u(\xi) d \xi \\
& -\frac{1}{2} \int_{0}^{L}\left[\int_{0}^{L} A^{2} g(x, \xi) \eta d \xi\right] \cdot \delta u(x) d x \\
= & \int_{0}^{L}\left[\int_{0}^{L} A^{2} g(x, \xi) \eta d \xi\right] \cdot \delta u(x) d x .
\end{aligned}
$$

Specifically, note that Eq. (20) can be derived based on the following relation:

$$
\begin{aligned}
\int_{0}^{L} & {\left[\int_{0}^{L} g(x, \xi) \eta d x\right] \delta u(\xi) d \xi } \\
& =\int_{0}^{L}\left[u(\xi) \int_{0}^{L} g(x, \xi) d x-\int_{0}^{L} g(x, \xi) u(x) d x\right] \delta u(\xi) d \xi \\
& =-\int_{0}^{L}\left[\int_{0}^{L} g(x, \xi) \eta d \xi\right] \delta u(x) d x \\
& =-\int_{0}^{L}\left[\int_{0}^{L} g(x, \xi) u(\xi) d \xi-u(x) \int_{0}^{L} g(x, \xi) d \xi\right] \delta u(x) d x,
\end{aligned}
$$

which holds due to symmetry of the function $g(x, \xi)$ with respect to the arguments $x$ and $\xi$, that is $g(x, \xi)=g(\xi, x)$. Based on Eqs. (19) and (20), Eq. (18) takes the form

$$
\begin{aligned}
\delta \Phi(u, \varepsilon, \eta)= & \int_{0}^{L}\left[-\bar{E} \frac{d^{2} u}{d x^{2}}+\int_{0}^{L} A \cdot g(x, \xi) \eta d \xi-f\right] A \cdot \delta u(x) d x \\
& -\left[F_{0}+\sigma_{l}(0) A\right] \delta u(0)-\left[F_{L}-\sigma_{l}(L) A\right] \delta u(L),
\end{aligned}
$$

where Eq. (7d) has been taken into account for $\sigma_{l}$ in Eq. (19). It is then readily seen that enforcing the stationarity condition $\delta \Phi(u, \varepsilon, \eta)=0$ leads to the equilibrium equation (10) and the mechanical (natural) boundary conditions (9).

\subsection{Total complementary energy functional}

The total complementary energy functional can be cast in the following form:

$$
\begin{aligned}
\Xi\left(\sigma_{l}, q\right)= & \frac{1}{2} \int_{0}^{L} \frac{A}{\bar{E}} \sigma_{l}^{2}(x) d x+\frac{1}{4} \int_{0}^{L} \int_{0}^{L} A^{2} \frac{q^{2}(x, \xi)}{g(x, \xi)} d x d \xi \\
& +\sigma_{l}(0) A \bar{u}_{0}-\sigma_{l}(L) A \bar{u}_{L} .
\end{aligned}
$$

In Eq. (23), the local stress $\sigma_{l}(x)$ and the long-range forces $A^{2} q(x, \xi) d x d \xi$ are statically admissible, that is they satisfy the equilibrium equation of the bar, Eq. (4). For generality, note that in Eq. (23) both the ends of the bar are assumed to be constrained, that is $u(0)=u_{0}$ and $u(L)=u_{L}$.

The first variation of $\Xi\left(\sigma_{l}, q\right)$ with respect to $\sigma_{l}$ and $q$ writes

$$
\begin{aligned}
\delta \Xi\left(\sigma_{l}, q\right)= & \int_{0}^{L} \frac{A \sigma_{l}}{\bar{E}} \cdot \delta \sigma_{l} d x+\frac{1}{2} \int_{0}^{L} \int_{0}^{L} A^{2} \frac{q(x, \xi)}{g(x, \xi)} \cdot \delta q d x d \xi \\
& +A u_{0} \cdot \delta \sigma_{l}(0)-A u_{L} \cdot \delta \sigma_{l}(L)
\end{aligned}
$$

For later convenience, the first variation $\delta \Xi$ in Eq. (24) may be also written as

$$
\begin{aligned}
\delta \Xi\left(\sigma_{l}, q\right)= & \int_{0}^{L} \frac{A \sigma_{l}}{\bar{E}} \cdot \delta \sigma_{l} d x+\frac{1}{2} \int_{0}^{L} \int_{0}^{L} A^{2} \frac{q(x, \xi)}{g(x, \xi)} \cdot \delta q d x d \xi \\
& +A u_{0} \cdot \delta \sigma_{l}(0)-A u_{L} \cdot \delta \sigma_{l}(L) \\
& -\left\{\int_{0}^{L} A \frac{d}{d x}\left[u(x) \cdot \delta \sigma_{l}\right] d x-A u(L) \cdot \delta \sigma_{l}(L)+A u(0) \cdot \delta \sigma_{l}(0)\right\} \\
& -\int_{0}^{L} \int_{0}^{L} A^{2}[u(x)+u(\xi)] \cdot \delta q d x d \xi .
\end{aligned}
$$

It is seen in fact that

$\int_{0}^{L} A \frac{d}{d x}\left[u(x) \cdot \delta \sigma_{l}\right] d x=A u(L) \cdot \delta \sigma_{l}(L)-A u(0) \cdot \delta \sigma_{l}(0)$

and

$\int_{0}^{L} \int_{0}^{L} A^{2}[u(x)+u(\xi)] \delta q d x d \xi=0$.

The latter equation may be readily demonstrated based on the constitutive law for the long-range forces, $q(x, \xi)=g(x, \xi)[u(\xi)-u(x)]$, and the symmetry of $g(x, \xi)=g(\xi, x)$ (in this regard, see also Eq. (21)).

Eq. (25) may be further simplified by noting that

$\int_{0}^{L} A \frac{d}{d x}\left[u(x) \cdot \delta \sigma_{l}\right] d x=\int_{0}^{L} A\left[\frac{d \delta \sigma_{l}}{d x} u(x)+\frac{d u(x)}{d x} \delta \sigma_{l}\right] d x$.

Based on Eqs. (4) and (6) it can be written that

$$
\begin{aligned}
\int_{0}^{L} & \int_{0}^{L} A^{2} u(x) \delta q d x d \xi \\
& =\int_{0}^{L} \int_{0}^{L} A^{2} u(x) g(x, \xi)[\delta u(\xi)-\delta u(x)] d x d \xi \\
& =\int_{0}^{L} A\left\{\int_{0}^{L} A g(x, \xi)[\delta u(\xi)-\delta u(x)] d \xi\right\} u(x) d x \\
& =\int_{0}^{L} A \frac{d \delta \sigma_{n l}(x)}{d x} u(x) d x .
\end{aligned}
$$

Also, based on Eq. (27)

$\int_{0}^{L} \int_{0}^{L} A^{2} u(\xi) \cdot \delta q d x d \xi=\frac{1}{2} \int_{0}^{L} \int_{0}^{L} A^{2}[u(\xi)-u(x)] \cdot \delta q d x d \xi$.

Due to Eqs. (28)-(30), taking into account the constitutive laws $\varepsilon=\sigma_{l} / E, \eta=q / g$, Eq. (25) may be then rewritten as 


$$
\begin{aligned}
\delta \Xi\left(\sigma_{l}, q\right)= & \int_{0}^{L} A\left(\varepsilon-\frac{d u}{d x}\right) \delta \sigma_{l} d x+\frac{1}{2} \int_{0}^{L} \int_{0}^{L} A^{2}\{\eta(x, \xi) \\
& -[u(\xi)-u(x)]\} \delta q d x d \xi-A\left[u(0)-u_{0}\right] \delta \sigma_{l}(0) \\
& +\left[u(L)-u_{L}\right] \delta \sigma_{l}(L)-\int_{0}^{L} A\left[\frac{d \delta \sigma_{l}}{d x}+\frac{d \delta \sigma_{n l}}{d x}\right] u(x) d x
\end{aligned}
$$

The latter term in Eq. (31) vanishes since $\delta \sigma_{l}$ and $\delta \sigma_{n l}$ are self-equilibrated variations in the class of statically admissible functions, that is

$\frac{d \delta \sigma_{l}}{d x}+\frac{d \delta \sigma_{n l}}{d x}=0$

herefore, it is seen that enforcing the stationarity condition $\delta \Xi\left(\sigma_{l}, q\right)=0$ for arbitrary $\delta \sigma_{l}$ and $\delta q$ leads to Eqs. (7b and c), along with the kinematic (essential) boundary conditions (8).

\subsection{Thermodynamic consistency}

Be $\dot{e}(x)$ the internal energy density rate of the elastic solid. In virtue of the first principle of thermodynamics in global form the following relation can be written:

$$
\begin{aligned}
\int_{V} \dot{e}(x) d V(x) & =A \int_{0}^{L} \dot{e}(x) d x \\
& =A \int_{0}^{L} f(x) \dot{u}(x) d x+\left[F_{L} \dot{u}_{L}-F_{0} \dot{u}_{0}\right]+A \int_{0}^{L}\left(r-\frac{d h}{d x}\right) d x,
\end{aligned}
$$

where $r$ is the heat source per unit volume and $h$ is the heat conduction. It has been shown by the virtual work principle that the following identity holds (Paola et al., 2009):

$$
\begin{aligned}
& A \int_{0}^{L} f(x) \dot{u}(x) d x+\left[F_{L} \dot{u}_{L}-F_{0} \dot{u}_{0}\right] \\
& \quad=A \int_{0}^{L} \sigma_{l}(x) \dot{\varepsilon}(x) d x+\frac{A^{2}}{2} \int_{0}^{L} \int_{0}^{L} q(x, \xi) \dot{\eta}(x, \xi) d \xi d x .
\end{aligned}
$$

Eq. (33) is the global version of the first thermodynamic principle. As already done in the literature for other models of non-local elasticity, a point-wise version of such principle can be provided also for the proposed model, specifically, in the following form:

$\dot{e}(x)=\sigma_{l}(x) \dot{\varepsilon}(x)+\frac{A}{2} \int_{0}^{L} q(x, \xi) \dot{\eta}(x, \xi) d \xi+\left(r-\frac{d h}{d x}\right)$.

Note that the non-local integral residual in Eq. (35) has a mechanical meaning since it represents half of the work done by the long-range forces for the relative displacements between interacting elements. In a thermodynamic context Eq. (35) shall be supplemented by the second principle of thermodynamics, that holds both in its global and local version. In global form the second principle of thermodynamics reads

$\int_{V} \dot{s}(x) d V(x)=A \int_{0}^{L} \dot{s}(x) d x \geqslant A \int_{0}^{L}\left[\frac{r}{T}-\frac{d}{d x}\left(\frac{h}{T}\right)\right] d x$,

where $\dot{s}$ represents the rate of the entropy density and $T$ is the absolute temperature. Eq. (36) may also be stated in strong, point-wise form stating that the rate of the entropy density must satisfy the Clausius-Duhem condition for irreversible local thermodynamic transformations, that is

$\dot{s} \geqslant \frac{r}{T}-\frac{d}{d x}\left(\frac{h}{T}\right)$

or, alternatively, by introducing the rate of the internal entropy density

$\dot{s}_{\text {int }}=\dot{s}-\left[\frac{r}{T}-\frac{d}{d x}\left(\frac{h}{T}\right)\right] \geqslant 0$.
The thermodynamic consistency of the proposed non-local bar can be assessed based on Eq. (38). To this purpose the Helmoltz free energy functional is introduced by means of a Legendre transform of the internal energy as $a=a(\varepsilon, \wp(\eta), T)=e(\varepsilon, \wp(\eta))-T s$ that accounts, separately, for the contributions of the local and the non-local elastic energies, i.e.

$e(\varepsilon, \wp(\eta))=e_{l}(\varepsilon)+\wp(\eta)=\frac{\bar{E} \varepsilon^{2}}{2}+\frac{A}{4} \int_{0}^{L} \eta^{2}(x, \xi) g(x, \xi) d \xi$,

where

$e_{l}(\varepsilon)=\frac{\bar{E} \varepsilon^{2}}{2} ; \quad \wp(\eta)=\frac{A}{2} \int_{0}^{L} e_{n l}(\eta) d \xi=\frac{A}{4} \int_{0}^{L} \eta^{2}(x, \xi) g(x, \xi) d \xi$.

Specifically, Eq. (40b) accounts for the non-local contribution to the elastic energy density, evaluated at the spatial coordinate $x$, provided by the long-range forces. Simple manipulations on Eq. (38) lead to the following relation:

$T \dot{s}_{\text {int }}=\dot{e}-\dot{a}-\dot{T} s-\left(r-\frac{d h}{d x}\right)+\Phi_{T} \geqslant 0$,

where $\Phi_{T}$ denotes the energy dissipation through thermal diffusion process and defined as

$\Phi_{T}=-\frac{h}{T} \frac{d T}{d x}$

Subsequently, taking into account Eq. (35), from Eq. (41) the Clausius-Duhem inequality is derived in the form

$T \dot{s}_{\text {int }}=\left[\sigma_{l}(x) \dot{\varepsilon}(x)+\frac{A}{2} \int_{0}^{L} q(x, \xi) \dot{\eta}(x, \xi) d \xi\right]-\dot{a}-\dot{T} s+\Phi_{T} \geqslant 0$.

Next, note that the first time-derivative $\dot{a}$ takes the form

$\dot{a}=\frac{\partial a}{\partial \varepsilon} \dot{\varepsilon}+\frac{\partial a}{\partial \wp(\eta)} \dot{\wp}(\eta)+\frac{\partial a}{\partial T} \dot{T}=\frac{\partial e}{\partial \varepsilon} \dot{\varepsilon}+\frac{\partial e}{\partial \wp(\eta)} \frac{\partial \wp(\eta)}{\partial \eta} \dot{\eta}+\frac{\partial a}{\partial T} \dot{T}$.

The point-wise form of the second thermodynamic principle must be satisfied for any thermomechanical process and henceforth also for an uniform temperature field, that is for $d T / d x=0$. Then, based on Eqs. (43) and (44), for $d T / d x=0$ it yields

$\left(\sigma_{l}-\frac{\partial e_{l}}{\partial \varepsilon}\right) \dot{\varepsilon}+\frac{A}{2} \int_{0}^{L}\left(q-\frac{\partial e_{n l}}{\partial \eta}\right) \dot{\eta} d \xi-\left(s+\frac{\partial a}{\partial T}\right) \dot{T} \geqslant 0$.

Eq. (45) must be fulfilled for any kind of thermodynamic transformation and, therefore, also for a reversible transformation corresponding to the equality sign in Eq. (45), that yields

$\sigma_{l}=\frac{\partial e_{l}}{\partial \varepsilon} ; \quad q=\frac{\partial e_{n l}}{\partial \eta} ; \quad s=-\frac{\partial a}{\partial T}$.

If Eq. (46) are assumed to hold also for the most general case $d T / d x \neq 0$, the following relation is obtained:

$\Phi_{T}=-\frac{h}{T} \frac{d T}{d x} \geqslant 0 \Rightarrow \frac{h}{T} \frac{d T}{d x} \leqslant 0$

reflecting the circumstance that the heat flux is directed toward negative gradients of the temperature. Eq. (46) are the state equations of the proposed non-local bar that, therefore, proves thermodynamically consistent since the state variables $\varepsilon(x)$ and $\eta(x, \xi)$ satisfy Eq. (46). In this context it is worth pointing out that the thermodynamic consistency of the proposed non-local bar holds regardless of the boundary conditions, thus leading to conclude that no thermodynamic restrictions hold on the boundary conditions (8) and (9), as in the classical local theory. 


\section{Approximate solutions of the elastic problem with long- range forces}

To the authors' best knowledge, no exact, closed-form solution can be found for the axial displacement field $u(x)$ of the elastic problem formulated in Section 2; only some approximate solutions based on the FDA (Paola et al., 2009) and the FFDA (Di Paola and Zingales, 2008) have been proposed. However such numerical solutions required, for accuracy reason, a very fine discretization grid thus leading to the need of developing alternative analytical approaches to the problem, as it will be pursued in the following sections. The solution strategies here proposed may be applied for long-range forces with both smooth and fractional decay, so that they may also represent a way to handle fractional differential equations (see also Podlubny, 1993).

\subsection{The Galerkin solution for the elastic continuum with long-range forces}

The Galerkin method to solve the elastic problem with longrange forces may be formulated by resorting to the principle of virtual displacements for the continuum mechanics with long range forces, which may be written as

$$
\begin{aligned}
\int_{0}^{L} & \delta u(x)\left[\frac{d \sigma}{d x}+f(x)\right] A d x+\left[F_{0}+\sigma_{l}(0) A\right] \delta u(0)+\left[F_{L}-\sigma_{l}(L) A\right] \delta u(L) \\
= & \int_{0}^{L} \delta u(x)\left\{\bar{E} \frac{d^{2} u}{d x^{2}}+A \int_{0}^{L} g(x, \xi)[u(\xi)-u(x)] d \xi+f(x)\right\} A d x \\
& +\left[F_{0}+\sigma_{l}(0) A\right] \delta u(0)+\left[F_{L}-\sigma_{l}(L) A\right] \delta u(L)=0,
\end{aligned}
$$

where $\delta u(x)$ is an arbitrary, but kinematically admissible, variation of the displacement field. Let us assume that the displacement field along the bar may be represented as

$u(x) \cong \varphi_{i}(x) c_{i} \quad(i=1,2, \ldots, m)$,

where $\varphi_{i}(x)(i=1,2, \ldots m)$ are trial, real-valued functions satisfying the kinematic boundary conditions and $c_{i}$ are real, unknown coefficients; also, the Einstein summation convention has been used for shortness. Replacing Eq. (49) for $u(x)$ in Eq. (48) yields

$$
\begin{aligned}
& \int_{0}^{L} \delta u(x)\left\{\bar{E} c_{i} \frac{d^{2} \varphi_{i}}{d x^{2}}+c_{i} A \int_{0}^{L} g(x, \xi)\left[\varphi_{i}(\xi)-\varphi_{i}(x)\right] d \xi+f(x)\right\} A d x \\
& +\left[F_{0}+\sigma_{l}(0) A\right] \delta u(0)+\left[F_{L}-\sigma_{l}(L) A\right] \delta u(L)=0 .
\end{aligned}
$$

The approximate form in Eq. (49) adopted for $u(x)$ does not allow to satisfy Eq. (50) for every choice of the variation $\delta u(x)$ so that the coefficients $c_{i}$ in Eq. (49) may be evaluated by requiring that the variations of the displacement field coincide with the trial functions, i.e. $\delta u(x)=\varphi_{j}(x)(j=1,2, \ldots, m)$. Eq. (50) may be further simplified by integration by parts of the first integral, which yields the following set of $m$ algebraic equations in the $m$ unknown coefficients $c_{i}$

$$
\begin{aligned}
& c_{i} \bar{E} A \int_{0}^{L} \frac{d \varphi_{j}}{d x} \frac{d \varphi_{i}}{d x} d x+c_{i} A^{2} \int_{0}^{L} \int_{0}^{L} g(x, \xi) \varphi_{j}(x)\left[\varphi_{i}(\xi)-\varphi_{i}(x)\right] d \xi d x \\
& \quad=\varphi_{j}(L) F_{L}+\varphi_{j}(0) F_{0}+\int_{0}^{L} f(x) \varphi_{j}(x) d x .
\end{aligned}
$$

The algebraic system of equations in Eq. (51) is cast in the matrix form

$\mathbf{K}^{(G)} \mathbf{c}=\left(\mathbf{K}^{(G-l)}+\mathbf{K}^{(G-n l)}\right) \mathbf{c}=\mathbf{F}$,

where $\mathbf{K}^{(G)}$ is the stiffness matrix evaluated via Galerkin approximation and the coefficient vector $\mathbf{c}^{T}=\left[c_{1}, c_{2}, \ldots, c_{m}\right]$.The load vector in Eq. (52) reads $F_{j}=\varphi_{j}(0) F_{0}+\varphi_{j}(L) F_{L}+\int_{0}^{L} f(x) \varphi_{j}(x) d x$ and the ele- ments of the local stiffness matrix $\mathbf{K}^{(G-l)}$ and the long-range interactions stiffness matrix $\mathbf{K}^{(G-n l)}$ are, respectively,

$k_{j i}^{(G-l)}=\bar{E} A \int_{0}^{L} \frac{d \varphi_{i}}{d x} \frac{d \varphi_{j}}{d x} d x$

$k_{j i}^{(G-n l)}=A^{2} \int_{0}^{L} \int_{0}^{L} g(x, \xi) \varphi_{j}(x)\left[\varphi_{i}(\xi)-\varphi_{i}(x)\right] d \xi d x$.

The global stiffness matrix $\mathbf{K}^{(G)}$ is symmetric (it is readily seen in this case since $\left.\delta u(x)=\varphi_{j}(x)(j=1,2, \ldots, m)\right)$ and positive definite. It may be then inverted to derive the coefficient vector c, Eq. (52), based on which the solution (49) may be computed.

\subsection{The Rayleigh-Ritz method for the elastic continuum with long- range forces}

Based on the total potential energy functional (17), next a weak formulation of the elastic equilibrium problem with long-range forces is proposed, as in the classical Rayleigh-Ritz method. It involves a series expansion similar to Eq. (49) as approximate solution for the displacement $u(x)$, where the trial functions $\varphi_{i}(x)$ shall satisfy the essential, kinematic, boundary conditions. Replacing Eq. (49) for $u(x)$ in Eq. (17) yields

$$
\begin{aligned}
\Phi(\mathbf{c})= & \frac{1}{2}\left\{\bar{E} A \int_{0}^{L}\left[c_{i} \frac{d \varphi_{i}(x)}{d x}\right]^{2} d x+\frac{A^{2}}{2} \int_{0}^{L} \int_{0}^{L} g(x, \xi)\left[c_{i} \tilde{\eta}_{i}(x, \xi)\right]^{2} d \xi d x\right\} \\
& -c_{i}\left[\varphi_{i}(0) F_{0}+\varphi_{i}(L) F_{L}+A \int_{0}^{L} f(x) \varphi_{i}(x) d x\right]
\end{aligned}
$$

where $\tilde{\eta}_{i}(x, \xi)=\varphi_{i}(\xi)-\varphi_{i}(x)$ and again the Einstein summation convention has been used. Eq. (54) is a quadratic functional of the unknown coefficients of the expansion in Eq. (49), which may be determined by minimizing the potential energy in Eq. (54) with respect to the coefficients $c_{i}$. This yields the system of algebraic equations

$\frac{\partial \Phi(\mathbf{c})}{\partial c_{i}}=0 ; \quad i=1,2, \ldots, m$

that can be written in compact form as

$\mathbf{K}^{(R)} \mathbf{c}=\left(\mathbf{K}^{(R-l)}+\mathbf{K}^{(R-n l)}\right) \mathbf{C}=\mathbf{F}$,

where $\mathbf{K}^{(R)}$ is the stiffness matrix evaluated via the Rayleigh-Ritz method. In Eq. (56) the elements of the local $\mathbf{K}^{(R-l)}$ and the non-local $\mathbf{K}^{(R-n l)}$ stiffness matrices are

$k_{i j}^{(R-l)}=\bar{E} A \int_{0}^{L} \frac{d \varphi_{i}}{d x} \frac{d \varphi_{j}}{d x} d x ;$
$k_{i j}^{(R-n l)}=\frac{A^{2}}{2} \int_{0}^{L} \int_{0}^{L} g(x, \xi) \tilde{\eta}_{i}(x, \xi) \tilde{\eta}_{j}(x, \xi) d \xi d x$.

As long as the same set of trial functions is used both in the Galerkin and the Rayleigh-Ritz method, it is readily seen that the elements of the local stiffness matrix $\mathbf{K}^{(R-l)}$ in Eq. (56) coincide with the corresponding elements of local stiffness matrix $\mathbf{K}^{(G-l)}$ in Eq. (52). Similarly, the elements of the non-local stiffness matrix $\mathbf{K}^{(R-n l)}$ in Eq. (56) may be recast exactly as the corresponding elements of the non-local stiffness matrix $\mathbf{K}^{(G-n l)}$ in Eq. (52). That is, the same set of trial functions in both the Galerkin and the Rayleigh-Ritz method yields the same solution for the elastic continuum with long-range forces, exactly as in classical mechanics. This leads to conclude that the used operators are self-adjoint and the introduced elastic potential energy is a convex functional of the state variables. In passing it is worth remarking that the Eringen model of non-local elasticity cannot lead to the same result, since the related potential 
energy function is not convex for a bounded domain and a generic attenuation function.

In the next section some numerical applications of the methods here discussed will be reported, for long-range forces with both smooth and fractional decay.

\section{Numerical applications}

Consider a uniform bar acted upon by two self-equilibrated point forces $F$ applied at the bar ends. As a first case, non-local effects are modelled based on long-range forces with the smooth exponential decay

$g(|x-\xi|)=C \exp (-|x-\xi| / \lambda), \quad C=\frac{\beta_{2} E}{2 A^{2} \lambda}$,

where $\lambda$ is the internal length, that is the influence distance beyond which the non-local effects may be neglected (Marotti De Sciarra, 2008). Two solutions are then built to the integro-differential equation (11): (i) by the Galerkin method or, equivalently, by the Rayleigh-Ritz method developed in Sections 4 and 5, where

$\varphi_{i}(x)=b^{-1 / 2} \sin (i \pi x / L), b=\int_{-L / 2}^{L / 2} \sin ^{2}(i \pi x / L) d x$

are taken as basis functions; (ii) by the FDA. Specifically, here the FDA involves a discretized version of Eq. (10) given as

$$
\begin{aligned}
\bar{E} A & \frac{u\left(x_{j+1}\right)-2 u\left(x_{j}\right)+u\left(x_{j-1}\right)}{\Delta x} \\
& +C \sum_{k=0, k \neq j}^{N}\left[u\left(x_{k}\right)-u\left(x_{j}\right)\right] \exp \left(-\left|x_{j}-x_{k}\right| / \lambda\right) A^{2} \Delta x^{2}=-f\left(x_{j}\right) A \Delta x,
\end{aligned}
$$

that can be cast in the matrix form

$\mathbf{K u}=\left(\mathbf{K}^{l}+\mathbf{K}^{n l}\right) \mathbf{u}=\mathbf{f}$,

where $\mathbf{f}^{T}=\Delta x\left[\begin{array}{llll}f\left(x_{1}\right) A & \cdots & \cdots & f\left(x_{m}\right) A\end{array}\right]$, and the stiffness matrices are

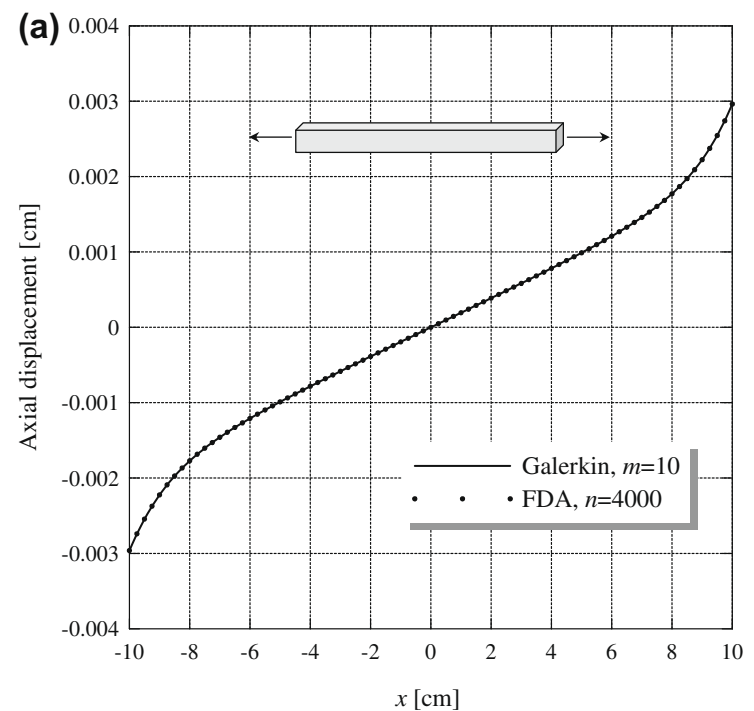

$\mathbf{K}^{l}=\left[\begin{array}{cccccc}K^{l} & -K^{l} & 0 & 0 & \ldots & 0 \\ & 2 K^{l} & -K^{l} & 0 & \ldots & 0 \\ & & \ldots & \ldots & \ldots & \ldots \\ & \mathrm{SYM} & & & 2 K^{l} & -K^{l} \\ & & & & & K^{l}\end{array}\right] ;$

$\mathbf{K}^{n l}=\left[\begin{array}{cccccc}K_{11}^{n l} & -K_{12}^{n l} & -K_{13}^{n l} & \cdots & \cdots & -K_{1 m}^{n l} \\ & K_{22}^{n l} & -K_{23}^{n l} & \cdots & \cdots & -K_{2 m}^{n l} \\ & & \cdots & \cdots & \cdots & \cdots \\ & \mathrm{SYM} & & & K_{m-1 m-1}^{n l} & -K_{m-1} m^{n l} \\ & & & & & K_{m m}^{n l}\end{array}\right]$

for $K^{l}=\bar{E} A / \Delta x$, for $K_{j j}^{n l}=\sum_{\substack{h=1 \\ h \neq j}}^{m} K_{j h}^{n l}$ and $K_{j h}^{n l}=C A^{2} \Delta x^{2} \exp \left(-\left|x_{j}-x_{h}\right| / \lambda\right)$.

That is, $\mathbf{K}^{l}$ and $\mathbf{K}^{n l}$ can be interpreted, respectively, as the local and non-local symmetric stiffness matrices of a consistent mechanical point-spring model, from which the continuous model (10) is derived as $\Delta x \rightarrow 0$ (see Paola et al., 2009 for details).

As a second case, non-local effects are modelled based on longrange forces with a fractional decay (12). Again, two solutions are built to Eq. (15): (i) by the Galerkin method or, equivalently, by the Rayleigh-Ritz method developed in Sections 4 and 5, where the basis functions (59) are used; (ii) by the FFDA (Shkanukov, 1996), used by Di Paola and Zingales (2008) to achieve faster convergence than by the standard FDA.

The Galerkin method, the FDA and the FFDA are all Fortrancoded and run on a Intel(R) Core(TM)2 Duo $2.10 \mathrm{GHz}$, where $0.001 \mathrm{~s}$ is the CPU time resolution. Results are presented here for the bar geometrical and mechanical parameters: $A=1 \mathrm{~cm}^{2}$, $E=2.1 \times 10^{6}$ da $\mathrm{N} \mathrm{cm}^{-2}, \quad L=20 \mathrm{~cm}, \quad F=10^{3}$ da N. Also, $\beta_{1}=\beta_{2}=0.5 ; \alpha=0.5$ and $c_{\alpha}=1.0 \mathrm{~cm}^{0.5}$ are selected in Eq. (12); $\lambda=2 \mathrm{~cm}$ in Eq. (58). Note that the numerical values for the above parameters are theoretical values chosen to enhance non-local effects in the response, case in which it appears more meaningful to assess the matching between the solution strategies proposed in the paper.

Fig. 2 shows the axial displacement for $g(|x-\xi|)$ given as Eqs. (12) and (58), respectively, when $m=10$ odd basis functions (59) are considered to build the Galerkin solution (i) and $n=4000$ volume elements $V_{j}$ are taken for the FDA and the FFDA solution. The latter is taken as benchmark solution since, as shown in Fig. 3, as

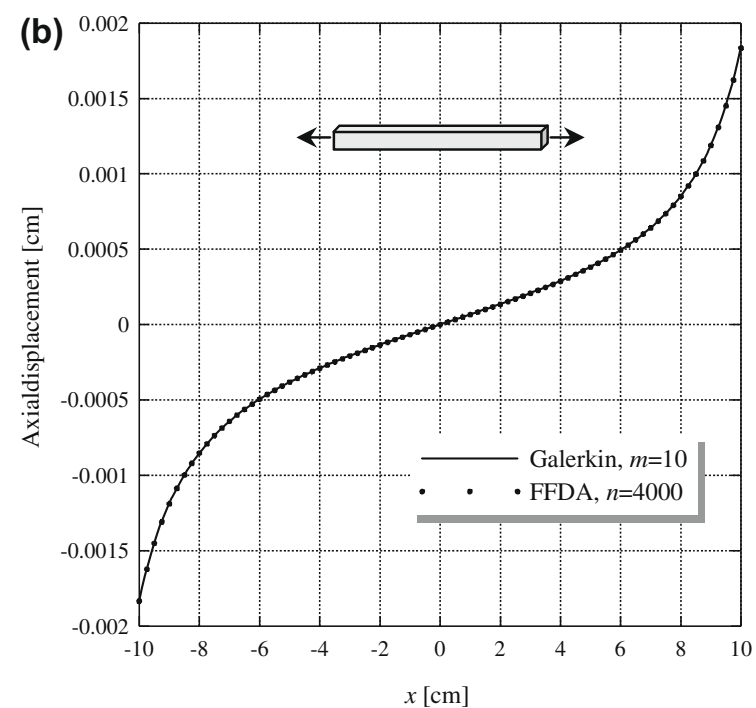

Fig. 2. Axial displacement in a uniform non-local bar in tension, for different distance-decaying long-range forces: (a) exponential decay; (b) fractional decay. 

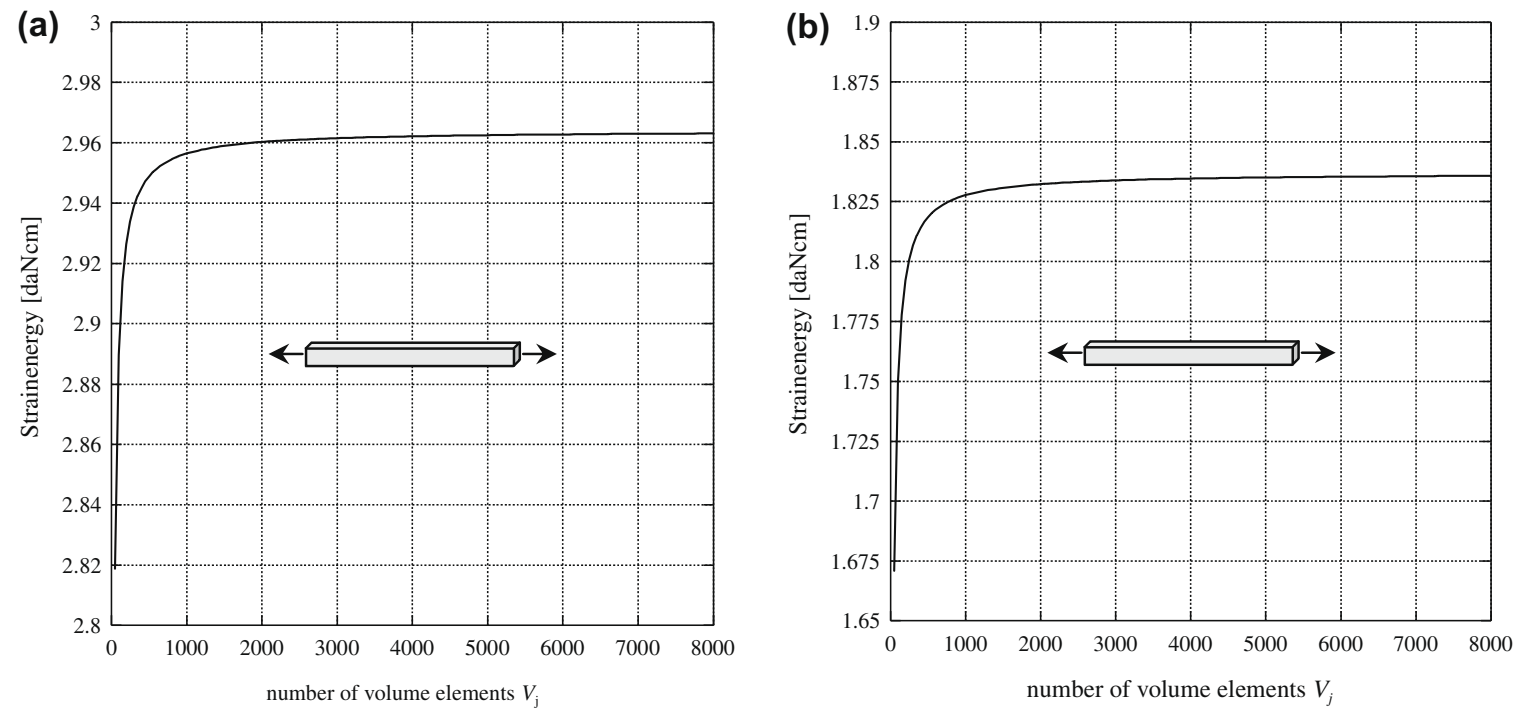

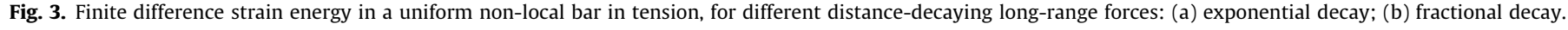
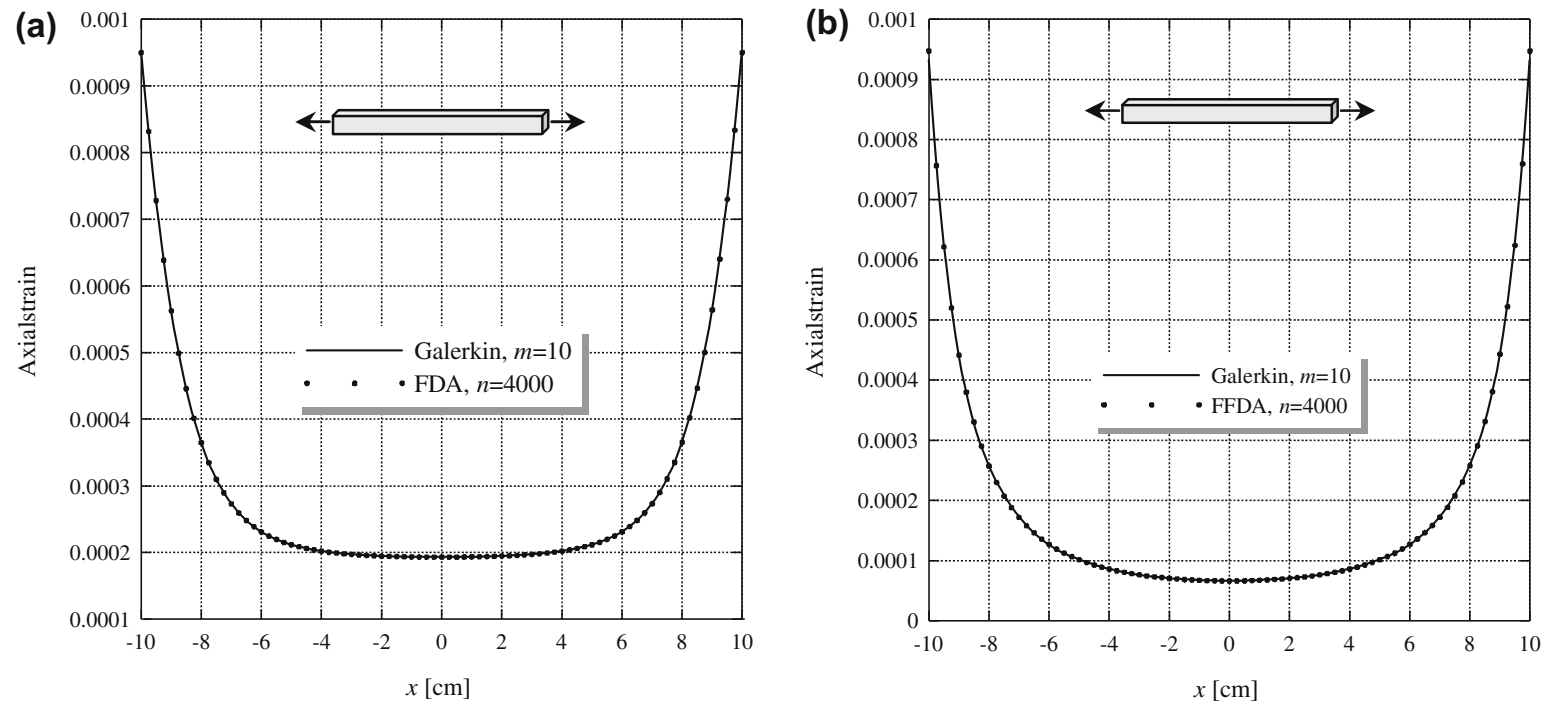

Fig. 4. Axial strain in a uniform non-local bar in tension, for different distance-decaying long-range forces: (a) exponential decay; (b) fractional decay.

$n \geqslant 4000$ no significant changes are encountered in the strain energy of the discrete model. A very good agreement is encountered between the Galerkin solution and, respectively, the FDA for exponential decay and the FFDA for fractional decay. However, the Galerkin solution proves more efficient. For long-range forces with exponential decay (58), in fact, the required CPU times are equal to $7.1 \mathrm{~s}$ for the Galerkin solution (i) and to $53.83 \mathrm{~s}$ for the FDA solution (ii). Further, for long-range forces with fractional decay (12), the required CPU times are equal to $20.9 \mathrm{~s}$ for the Galerkin solution (i) and to $52.51 \mathrm{~s}$ for the FFDA solution. Note finally that both Fig. 3a and b shows an almost linear displacement function in a central core of the bar, the size of the core being strictly influenced by the values of internal length $\lambda$ and $c_{\alpha}$. At the limit $\lambda, c_{\alpha} \rightarrow 0$ the displacement field is linear throughout the whole bar with vanishing non-local effects.

Based on the computed axial displacement, all the response variables may be derived. For instance, the axial strain is shown in Fig. 4. The values attained at the bar ends are equal to the local contribution only, $\varepsilon=F /(\bar{E} A)=9.52 \times 10^{-4}$; this is consistent with Eq. (9), where $\sigma_{n l} A=0$ at the bar ends.
It is finally worth remarking that, for $n>4000$, displacement and strain responses do differ but not significantly from the displacement and the strain responses obtained for $n=4000$ and reported in Figs. 2 and 4. For shortness and to avoid almost superposing lines, they have not been included in Figs. 2 and 4.

\section{Concluding remarks}

It has been shown that classical methods of linear elasticity theory, such as the Galerkin method and the Rayleigh-Ritz method, can be readily applied to solve a $1 \mathrm{D}$ non-local bar recently proposed in the literature. In this manner accurate approximate solutions have been obtained, with a significant reduction of computational effort as compared to the numerical solutions previously built (Di Paola and Zingales, 2008; Di Paola et al., 2009).

It is worth remarking that the non-local elasticity model, here formulated and solved for a 1D bar, lends itself to a straightforward generalization to 3-D continua, based on the same concepts. Specifically, the $3 \mathrm{D}$ formulation will be pursued by modelling the 
long-range force between two volume elements as a force depending on the relative displacement measured along the line connecting the two volume elements in the original configuration. Such 3D non-local forces, under the assumption of small displacements, prove invariant with respect to rigid-body motions, including rigid-body rotations, thus ensuring the consistency of the 3D non-local elasticity model. A detailed formulation and pertinent numerical results will be presented in future self-contained studies.

\section{Appendix A}

Some interesting conclusions may be drawn if a comparison is sought between the proposed model of non-local bar and the one based on the well-known Eringen theory (Eringen, 1972). For this purpose it is here reminded that the Eringen theory of non-local elasticity involves a constitutive law with a weighted average of the strain field given as

$\sigma(x)=\bar{E} \varepsilon(x)+\beta_{2} \int_{D} \tilde{g}(|x-\xi|) \varepsilon(\xi) d \xi$,

where $\tilde{g}(|x-\xi|)$ is the attenuation function. Specifically, assume that the attenuation function is given as $\tilde{g}(|x-\xi|)=\widetilde{C} \exp (-|x-\xi| / \lambda)$. Substituting Eq. (A.1) for $\sigma(x)$ in the equilibrium equation $d \sigma(x) / d x=-f(x)$ yields, after some manipulations, the following governing equation:

$$
\begin{aligned}
& \frac{d^{2} u(x)}{d x^{2}}+\frac{\beta_{2} \widetilde{C}}{\lambda^{2} \bar{E}} \int_{D}[u(\xi)-u(x)] \exp (-|x-\xi| / \lambda) d \xi \\
& -\frac{\beta_{2} \widetilde{C}}{\lambda \bar{E}}\left\{\left[u(x)-u\left(D^{+}\right)\right] e^{-\left(D^{+}-x\right) / \lambda}+\left[u(x)-u\left(D^{-}\right)\right] e^{-\left(x-D^{-}\right) / \lambda}\right\} \\
& =-\frac{f(x)}{\bar{E}}
\end{aligned}
$$

where $D^{-}$and $D^{+}$are, respectively, the left and right end of the bar domain. For an infinite bar, Eq. (A.2) is identical to Eq. (11) as long as $\widetilde{C}=C A \lambda^{2} / \beta_{2}$ and is taken. That is, for exponentially-decaying long-range forces, the proposed mechanical model is indeed the mechanical model underlying the Eringen constitutive law. The same equivalence can be demonstrated for the fractional model of long-range forces (Cottone et al., 2009; Di Paola and Zingales, 2008).

If the bar is finite, however, Eqs (10) and (A.2) are not identical but differ for the last two terms in the 1.h.s. of Eq. (A.2). This difference allows to point out some mechanical inconsistencies of the Eringen constitutive law that can be described by rewriting Eq. (A.2) in a finite difference form as in Eq. (60). This yields

$\mathbf{K u}=\left(\mathbf{K}^{l}+\mathbf{K}^{n l}+\mathbf{K}^{r}\right) \mathbf{u}=\mathbf{f}$,

where the additional residual matrix $\mathbf{K}^{r}$ is written as

$$
\mathbf{K}^{r}=\frac{\beta_{2} \widetilde{C} A \cdot \Delta x}{\lambda}\left[\begin{array}{ccccc}
e^{-L / \lambda} & 0 & \cdots & 0 & -e^{-L / \lambda} \\
-e^{-x_{2} / \lambda} & \left(e^{-x_{2} / \lambda}+e^{-\left(L-x_{2}\right) / \lambda}\right) & \cdots & 0 & -e^{-\left(L-x_{2}\right) / \lambda} \\
\cdots & \cdots & \cdots & \cdots & \cdots \\
-e^{-L / \lambda} & 0 & \cdots & 0 & e^{-L / \lambda}
\end{array}\right] .
$$

The latter is not symmetric thus leading to conclude that no consistent mechanical model may be associated to the non-local elasticity model described by Eq. (A.1). In this regard, note that the same conclusions have been previously drawn by several authors (Borino et al., 2003; Marotti De Sciarra, 2008), who have used a properly modified attenuation function to attain symmetry of the considered matrices.

\section{References}

Aifantis, E.C., 1994. Gradient effects at macro micro and nano scales. Journal of the Mechanical Behavior of Materials 5, 355-375.

Andrianov, I.V., Awrejcewicz, J., 2005. Continuous models for 1D discrete media valid for higher-frequency domain. Physics Letters A 345, 55-62.

Borino, G., Failla, B., Parrinello, F., 2003. A symmetric non-local damage theory. International Journal of Solids and Structures 40, 3621-3645.

Cottone, G., Di Paola, M., Zingales, M., 2009. Fractional mechanical model for the dynamics of non-local continuum. In: Mastorakis, N., Sakellaris, J. (Eds.), Advances in Numerical Methods. Lecture Notes in Electrical Engineering, vol. 11. Springer-Verlag.

Di Paola, M., Zingales, M., 2008. Long-range cohesive interactions of non-local continuum faced by fractional calculus. International Journal of Solids and Structures 45 (21), 5642-5659.

Di Paola, M., Failla, G., Zingales, M., 2009. Physically-based approach to the mechanics of strong non-local linear elasticity theory. Journal of Elasticity, doi:10.1007/s10659-009-9211-7.

Eringen, A.C., 1972. Linear theory of non-local elasticity and dispersion of plane waves. International Journal of Engineering Science 10, 425-435.

Fuschi, P., Pisano, A.A., 2003. Closed form solution for a nonlocal elastic bar in tension. International Journal of Solids and Structures 40,13-23.

Kroner, E., 1967. Elasticity theory of materials with long-range cohesive forces. International Journal of Solids and Structures 3, 731-742.

Laskin, N., Zaslavsky, G., 2006. Nonlinear fractional dynamics on a lattice with long range interactions. Physica A: Statistical Mechanics and its Applications 368, 38-54.

Marotti De Sciarra, F., 2008. Variational formulations and a consistent finiteelement procedure for a class of non-local elastic continua. International Journal of Solids and Structures 45, 4184-4202.

Metrikine, A.V., Askes, H., 2002a. One-dimensional dynamically consistent gradient elasticity models derived from a discrete microstructure. Part 1: Generic formulation. European Journal of Mechanics A/Solids 21, 555-572.

Metrikine, A.V., Askes, H., 2002b. One-dimensional dynamically consistent gradient elasticity models derived from a discrete microstructure. Part 2: Static and dynamic response. European Journal of Mechanics A/Solids 21, 573-588.

Podlubny, I., 1993. Fractional Differential Equations. Academic Press, New-York.

Polizzotto, C., 2001. Nonlocal elasticity and related variational principles. International Journal of Solids and Structures 38, 7359-7380.

Polizzotto, C., 2003. Gradient elasticity and non standard boundary conditions. International Journal of Solids and Structures 40, 7399-7423.

Samko, S.G., Kilbas, A.A., Marichev, O.I., 1988. Fractional Integral and Derivatives. Gordon \& Breach, Amsterdam.

Silling, S.A., 2000. Reformulation of elasticity theory for discontinuities and longrange forces. Journal of Mechanics and Physics of Solids 48, 175-209.

Silling, S.A., Zimmermann, M., Abeyaratne, R., 2003. Deformation of a peridynamic bar. Journal of Elasticity 73, 173-190.

Shkanukov, M.K., 1996. On the convergence of difference schemes for differential equations with a fractional derivative. Doklady Akademii Nauk 348, 746-748. 